\title{
Correction to: Longitudinal FRET Imaging of Glucose and Lactate Dynamics and Response to Therapy in Breast Cancer Cells
}

Jianchen Yang ${ }^{1}$, Tessa Davis ${ }^{1}$, Anum S. Kazerouni ${ }^{1}$, Yuan-I. Chen ${ }^{1}$, Meghan J. Bloom ${ }^{1}$, Hsin-Chih Yeh ${ }^{1,2}$, Thomas E. Yankeelov ${ }^{1,3,4,5,6,7}$, and John Virostko $3,4,6$

${ }^{1}$ Department of Biomedical Engineering, The University of Texas At Austin, Austin, TX 78712, USA

${ }^{2}$ Texas Materials Institute, The University of Texas At Austin, Austin, TX 78712, USA

${ }^{3}$ Department of Diagnostic Medicine, The University of Texas At Austin, 201 E. 24th Street, 1 University Station (C0200), Austin, TX 78712, USA

${ }^{4}$ Department of Oncology, The University of Texas At Austin, Austin, TX 78712, USA

${ }^{5}$ Oden Institute for Computational Engineering and Sciences, The University of Texas At Austin, Austin, TX 78712, USA

${ }^{6}$ Livestrong Cancer Institutes, The University of Texas At Austin, Austin, TX 78712, USA

${ }^{7}$ Department of Imaging Physics, The University of Texas MD Anderson Cancer Center, Houston, TX 77030, USA 2021

\section{Correction to: Mol Imaging Biol}

https://doi.org/10.1007/s11307-021-01639-4

This article was updated to correct the Funding information.

Publisher's Note Springer Nature remains neutral with regard to jurisdictional claims in published maps and institutional affiliations.

Correspondence to: John Virostko; e-mail: jack.virostko@ austin.utexas.edu 